\title{
Hoinga: a supernova remnant discovered in the SRG/eROSITA All-Sky Survey eRASS1
}

\author{
W. Becker ${ }^{1,2}$, N. Hurley-Walker ${ }^{3}$, Ch. Weinberger ${ }^{1}$, L. Nicastro ${ }^{4}$, M. G. F. Mayer ${ }^{1}$, A. Merloni ${ }^{1}$, and J. Sanders ${ }^{1}$ \\ ${ }^{1}$ Max-Planck-Institut für extraterrestrische Physik, Giessenbachstraße, 85748 Garching, Germany \\ e-mail: web@mpe.mpg.de \\ 2 Max-Planck-Institut für Radioastronomie, Auf dem Hügel 69, 53121 Bonn, Germany \\ ${ }^{3}$ International Centre for Radio Astronomy Research, Curtin University, Bentley WA 6102, Australia \\ ${ }^{4}$ INAF - Osservatorio di Astrofisica e Scienza dello Spazio di Bologna, Via Piero Gobetti 93/3, 40129 Bologna, Italy
}

Received 17 December 2020 / Accepted 12 February 2021

\begin{abstract}
Supernova remnants (SNRs) are observable for about $(6-15) \times 10^{4} \mathrm{yr}$ before they fade into the Galactic interstellar medium. With a Galactic supernova rate of approximately two per century, we can expect to have of the order of 1200 SNRs in our Galaxy. However, only about 300 of them are known to date, with the majority having been discovered in Galactic plane radio surveys. Given that these SNRs represent the brightest tail of the distribution and are mostly located close to the plane, they are not representative of the complete sample. The launch of the Russian-German observatory SRG/eROSITA in July 2019 brought a promising new opportunity to explore the Universe. Here we report findings from the search for new SNRs in the eROSITA all-sky survey data which led to the detection of one of the largest SNRs discovered at wavelengths other than the radio: G249.5+24.5. This source is located at a relatively high Galactic latitude, where SNRs are not usually expected to be found. The remnant, 'Hoinga', has a diameter of about 4.4 and shows a circular shaped morphology with diffuse X-ray emission filling almost the entire remnant. Spectral analysis of the remnant emission reveals that an APEC spectrum from collisionally ionised diffuse gas and a plane-parallel shock plasma model with non-equilibrium ionisation are both able to provide an adequate description of the data, suggesting a gas temperature of the order of $\mathrm{kT}=0.1_{-0.02}^{+0.02} \mathrm{keV}$ and an absorbing column density of $N_{\mathrm{H}}=3.6_{-0.6}^{+0.7} \times 10^{20} \mathrm{~cm}^{-2}$. Various X-ray point sources are found to be located within the remnant boundary but none seem to be associated with the remnant itself. Subsequent searches for a radio counterpart of the Hoinga remnant identified its radio emission in archival data from the Continuum HI Parkes All-Sky Survey and the 408-MHz 'Haslam' all-sky survey. The radio spectral index $\alpha=-0.69 \pm 0.08$ obtained from these data definitely confirms the SNR nature of Hoinga. We also analysed INTEGRAL SPI data for fingerprints of ${ }^{44} \mathrm{Ti}$ emission, which is an ideal candidate with which to study nucleosynthesis imprinting in young SNRs. Although no ${ }^{44} \mathrm{Ti}$ emission from Hoinga was detected, we were able to set a $3 \sigma$ upper flux limit of $9.2 \times 10^{-5} \mathrm{ph} \mathrm{cm}^{-2} \mathrm{~s}^{-1}$. From its size and X-ray and radio spectral properties we conclude that Hoinga is a middle-aged Vela-like SNR located at a distance of about twice that of the Vela SNR, i.e. at $\sim 500$ pc.
\end{abstract}

Key words. supernovae: general - supernovae: individual: Hoinga (G249.5+24.5)

\section{Introduction}

A long series of observations have taught astronomers that there are many different types of stars. Findings in atomic and nuclear physics have made it possible to understand the development of these stars over the past few decades. According to this, the fate of a star at the end of its thermonuclear evolution essentially depends on only one parameter: the mass of a star decides whether its death is gentle or violent. More massive stars with $M \geq 8 M_{\odot}$ end their lives with a supernova ( $\mathrm{SN}$ ) explosion, which is not only often associated with the formation of other exotic star types such as neutron stars (NSs) or black holes, but also represents a new beginning of stellar evolution by enrichment and decompression of the surrounding interstellar medium. A prominent example for this is the Solar System itself which shows imprints in metal abundance of a past SN which took place 4.567 Gyr ago (Gritschneder et al. 2012).

Supernovae are considered to be rare events which happen in our Milky Way on average every $30-50$ yr (e.g. Keane \& Kramer 2008), though no SN event has been directly observed in our Galaxy in the past $400 \mathrm{yr}$. Indeed, in the past two millennia, only seven Galactic SN are the subject of historical records: SN 185 (RCW 86), SN 386 (G11.2-0.3), SN 1006, SN 1054 (Crab), SN 1181 (3C58), SN 1572 (Tycho), and SN 1604 (Kepler); see also Stephenson (2017) and references therein. However, there are additional promising candidates discussed in the literature, such as for example CAS A (Green \& Stephenson 2017) and Vela-Jr (Aschenbach 1998).

Certainly, visible-band extinction of the SN emission and its distance to Earth plays a crucial role when it comes to recognising a $\mathrm{SN}$ with the naked eye. A prominent example of this effect is demonstrated by the missing reports of the CAS A SN event which is believed to have taken place about $300 \mathrm{yr}$ ago. No widespread reports of CAS A exist in the literature of the 17th century (cf. Hartmann et al. 1997). A more recent example of an unrecognised SN is that of the youngest SN known in our Galaxy, G1.9+0.3, which was completely missed by optical observatories about 100 yr ago (Reynolds et al. 2008).

In contrast to $\mathrm{SNe}$ which are only observable on a timescale of months to years, their remnants (SNRs) are detectable over a 
large range of the electromagnetic bands for more than 60000 150000 yr. However, today only about 300 SNRe are known (cf. Green 2019), most of which were discovered in Galactic plane radio surveys. Assuming that the radio lifetime of a SNR bright enough to be detected with current radio telescopes is at least about $60 \mathrm{kyr}$ (Frail et al. 1994), there is a discrepancy by a factor of between four and six between the observed and expected number of SNRs. Even if one takes into account the fact that very massive stars may form a black hole without a luminous SN (e.g. Kochanek et al. 2008; Adams et al. 2017) there is still a significant mismatch between the expected and known number of SNRs. The discrepancy is possibly explained by the fact that the radio sample of SNRs is not complete. Reasons that may prevent a radio bright remnant from being detected in radio surveys are various:

- A SN shock wave may expand within the hot phase of the ISM and reach a very large diameter until it has swept up sufficient mass from the low-density gas to form a radio shell. Density inhomogeneities in such a large volume will cause distortions in the shell and can make the identification as a SNR rather difficult, in particular in the presence of confusing unrelated emission from other nearby sources in the same region of the sky.

- A SN shock wave may expand in a very dense medium, making the SNR lifetime rather short, because material is quickly swept up and decelerated. Such an environment is likely to be relevant for example for massive star members of OB-associations that are surrounded by dense molecular clouds and warm gas. Even during their short lifetime, such events are difficult to identify within the strong thermal radio emission from those regions.

- There is a strong bias towards bright resolved objects in observations towards the inner Galaxy.

- Low-surface-brightness SNRs are easily missed in radio surveys if they are below the sensitivity limits of the surveys or if they are confused with other objects in the same area.

- Old SNRs which are in the phase of dissolving into the ISM may have incomplete radio shells that may then prevent these sources from being identified as SNRs.

- SNRs located away from the Galactic plane are easily missed in radio surveys, as this area is where these events are typically targeted.

Given these selection effects in radio surveys and the detection of unknown SNRs in previous X-ray surveys (e.g. Pfeffermann \& Aschenbach 1996; Busser et al. 1996; Asaoka \& Aschenbach 1994; Asaoka et al. 1996; Egger et al. 1996; Folgheraiter et al. 1996), as well as the detection of more than 70 highly significant SNR candidates in our analysis of the ROSAT All-Sky-Survey data, it was deemed worthy to start searching for undiscovered SNRs in the first eROSITA All-Sky Survey RASS1 (Predehl et al. 2021).

In this paper we report the discovery of the SNR G249.5+24.5 in the eROSITA data. With a diameter of about 4.4 it is among the largest SNRs discovered at wavelengths other than the radio. The structure of the paper is as follows: eROSITA and ROSAT observations of the remnant along with the data analysis are described in Sect. 2. In Sect. 3 we describe the analysis of archival radio data taken from the Continuum HI Parkes All-Sky Survey (CHIPASS) and the $408 \mathrm{MHz}$ all-sky continuum survey in order to search for a radio counterpart to the remnant and to explore its radio emission properties. Section 4 presents our exploration of spectrometer data from INTEGRAL in order to search for possible ${ }^{44} \mathrm{Ti}$ decay radiation associated with the remnant. In Sect. 5 we summarise and discuss our results.

\section{X-ray observations and data analysis}

\subsection{Hoinga in the eROSITA All-Sky Survey}

The German-built X-ray telescope eROSITA (extended Röntgen Survey Imaging Telescope Array) is one of two instruments on the Russian-German observatory SRG (Spectrum RöntgenGamma; Sunyaev et al., in prep.). eROSITA consists of seven aligned X-ray telescopes (TM1-TM7), each nested with 54 gold-coated mirror shells which have a focal length of $1600 \mathrm{~mm}$. All telescopes observe the same sky-region simultaneously in the $0.2-8 \mathrm{keV}$ band-pass though each focuses the collected $\mathrm{X}$ rays on its own pn-CCD camera (Meidinger et al. 2014). The latter is an improved version of the pn-CCD camera aboard XMM-Newton (Strüder et al. 2001). eROSITA has a spectral resolution of $\sim 70 \mathrm{eV}$ at $1 \mathrm{keV}$ and a temporal resolution of $50 \mathrm{~ms}$. Its field of view (FOV) is $1^{\circ}$. The on-axis effective area of all seven telescopes combined is slightly higher than that of the XMM-Newton pn + MOS cameras in the key $0.5-2.0 \mathrm{keV}$ band-pass. In pointing mode (on axis) the angular resolution of eROSITA is $18^{\prime \prime}$ (HEW) whereas in survey mode it is $26^{\prime \prime}$ (FOV averaged). Source location accuracy is of the order of 4.'5 $(1 \sigma)$. The second instrument onboard SRG is the Russian X-ray concentrator MIKHAIL PAVLINSKY ART-XC (Astronomical Röntgen Telescope - X-ray Concentrator) (Pavlinsky et al. 2018), which is sensitive in the hard X-ray band from 4 up to $30 \mathrm{keV}$, making it complementary to the eROSITA soft band.

The SRG was launched into an L2 orbit on July 13, 2019, with a Russian Proton-M launch vehicle. After a three-month calibration and science verification phase it started its first all-sky survey on December 13, 2019. With a scan rate of $0.025 \mathrm{deg} \mathrm{s}^{-1}$, a spacecraft revolution duration of $4 \mathrm{~h}$ and a central FOV passage time of about 40 s (Predehl et al. 2021), each survey takes 6 months to complete. eROSITA is supposed to take eight all-sky surveys over a time period of $4 \mathrm{yr}$.

The X-ray data we report here were taken during the first eROSITA all-sky survey eRASS1, completed on June 12, 2020. As the main science driver of the SGR mission is to explore the nature of dark energy, its orbit was chosen so that the Ecliptic poles get the deepest exposure, leading to an exposure of the Galactic plane which is of the order of $\sim 200-300$ seconds per survey.

First results of eRASS1, including a fascinating, detail-rich, three-energy-band colour-coded image of the $0.3-8.0 \mathrm{keV}$ X-ray sky, were recently released. The survey represents the deepest view of the whole X-ray sky today and led to the discovery of the large-scale symmetric hot-gas structures in the Milky Way halo, called 'eROSITA Bubbles' (Predehl et al. 2020), among many other exciting results.

Searching this survey map for unknown extended sources revealed the existence of a new SNR at Galactic coordinates $l=249^{\circ} .5$ and $b=24.5$, labelled G249.5+24.5 which we dub Hoinga ${ }^{1}$. Figure 1 depicts a colour-coded image of the relevant sky region which shows Hoinga with its neighbours the Antlia Loop and the Vela SNR.

The data we use in our analysis were processed by the eSASS (eROSITA Standard Analysis Software) pipeline and have the processing number \#946. For the data analysis we used eSASS version 201009 (released on October 9th, 2020) ${ }^{2}$. Within the eSASS pipeline, X-ray data of the eRASS sky are divided into

\footnotetext{
1 In honor of the first author's hometown Bad Hönningen am Rhein: Hoinga was its medieval name.

2 cf.https://erosita.mpe.mpg.de/
} 


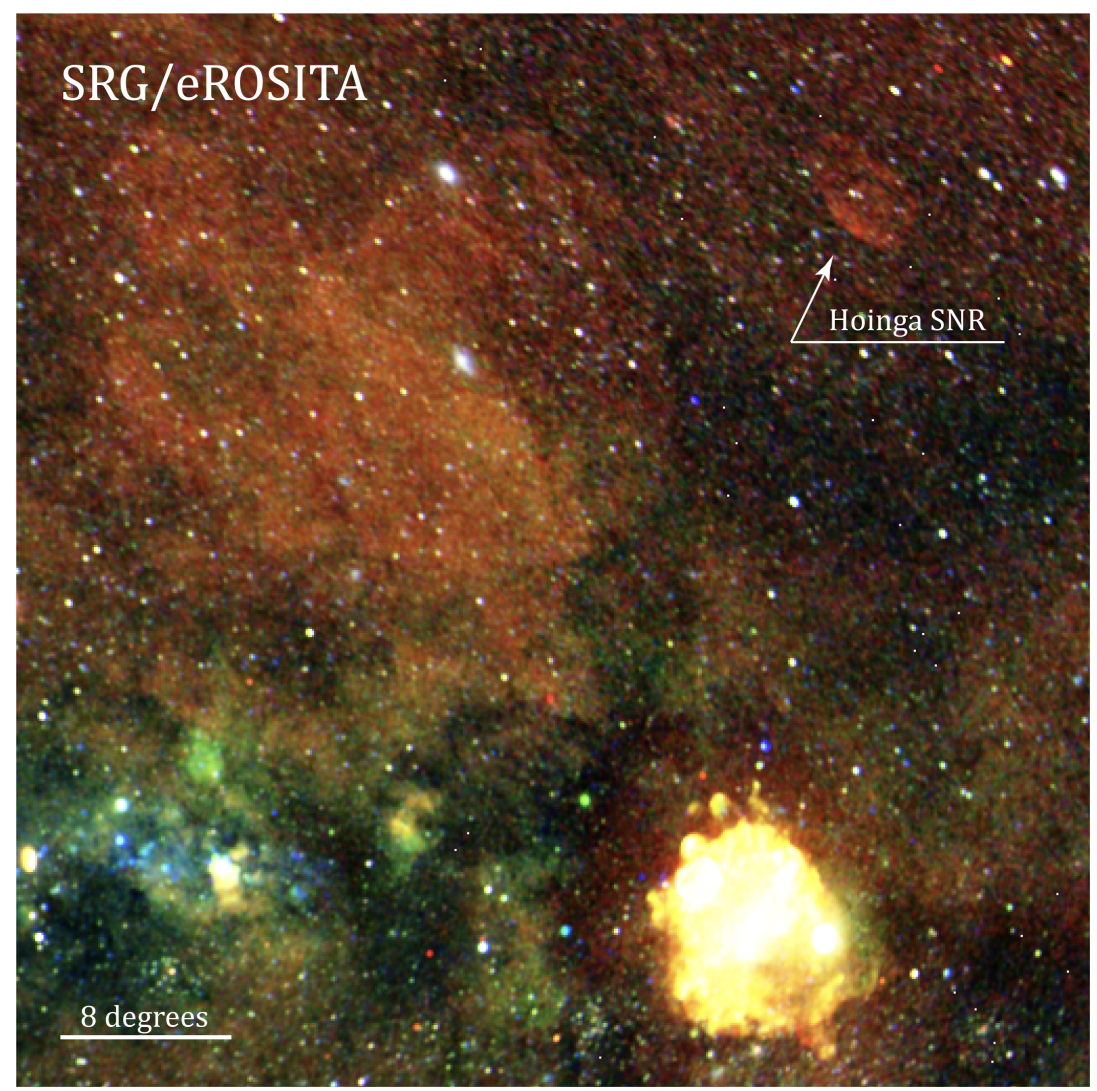

Fig. 1. Cutout of the SRG/eROSITA all-sky survey image from eRASS1 data. The image shows, among many other sources, the extended X-ray emission from the $24^{\circ}$ diameter large Antlia Loop in its upper left quadrant and the emission from the Vela SNR in its lower right. The emission from the Hoinga SNR in the upper right quadrant of the image is indicated. The image is an Aitoff projection of photons that have been colour-coded according to their energy (red for energies $0.3-0.6 \mathrm{keV}$, green for $0.6-1 \mathrm{keV}$, blue for $1-2.3 \mathrm{keV}$ ). The image was smoothed with a $10^{\prime}$ FWHM Gaussian filter.
4700 partly overlapping sky tiles of $3.6 \times 3^{\circ} .6$ each. These are numbered using six digits, three for RA and three for Dec, representing the sky tile centre position in degrees. The majority of Hoinga's emission falls into the eRASS1 sky tiles numbered 142108, 146108, and 143105 whereas the six surrounding sky tiles $(145111,142111,139111,139108,146105,140105)$ needed to be included for complete coverage of the remnant. Hoinga was observed in eRASS1 between 15 and 22 May, 2020, in a total of 29 telescope passages, resulting in an unvignetted averaged exposure time of approximately $240 \mathrm{~s}$.

Figure 2 shows an RGB image of the remnant which has been colour coded according to the energy of the detected photons. To produce the image, we first created images for the three energy bands $0.2-0.7,0.7-1.2$, and $1.2-2.4 \mathrm{keV}$, respectively. The spatial binning in these images was set to $26^{\prime \prime}$ which reflects eROSITA's FOV averaged angular resolution in survey mode. Data from all seven telescopes were used as we did not notice a significant impact of the light leak in TM5 and TM7. In order to enhance the visibility of Hoinga's diffuse emission in these images whilst leaving point sources unsmoothed to the greatest possible extent we applied the adaptive kernel smoothing algorithm of Ebeling et al. (2006) with a Gaussian kernel of $4.5 \sigma$.

The image analysis clearly reveals that Hoinga's X-ray emission is very soft. The majority of its emission is detected in the $0.2-0.7 \mathrm{keV}$ band, leaving the remnant undetected in eRASS 1 above $0.7 \mathrm{keV}$. The shape of Hoinga appears largely circular except for the remnant's west side for which no emission is detected by eROSITA. The morphological structure of the remnant is clearly centre-filled without a distinct shellbrightening structure. However, its soft X-ray emission slightly brightens towards the southern direction with a knot-like structure (cf. also Fig. 3). We will explore this region in more detail when additional eROSITA data become available. To determine the geometrical centre of the remnant we fitted an annulus to the outer boundary of its X-ray emission. In right ascension and declination the remnant centre is then found to be at RA = 09:31:53.47, Dec $=-17: 01: 36.7$ (J2000), which according to the eROSITA naming convention assigns it the catalogue name 1eRASS J093153.47-170 136.7.

\subsection{Hoinga in the ROSAT All-Sky Survey}

After the discovery of Hoinga in eRASS1 data we went back to the archival ROSAT all-sky survey (RASS) to check whether the remnant was detected. The ROSAT RASS was performed between June 1990 and August 1991, almost exactly $30 \mathrm{yr}$ before eRASS1. The ROSAT PSPC (position-sensitive proportional counter), which was in the focal plane during the survey, was sensitive in the 0.1-2.4 keV energy range (Pfeffermann et al. 2003). The angular resolution in the survey was $45^{\prime \prime}$. RASS data are divided into 1378 partly overlapping sky tiles, each covering $6.4 \times 6.4$ of the sky. Hoinga is located in the RASS data with the sequence numbers 932025, 932026. It was observed between November 11-18, 1990. After applying the standard ROSAT data processing using the Extended Scientific Analysis Software EXSAS (Zimmermann et al. 1994), we created images from the photons in the $0.1-0.7,0.7-1.2$, and $1.2-2.4 \mathrm{keV}$ energy bands. While there is no emission seen in the medium and hard bands, the soft-band image clearly shows a hint of circular shaped soft X-ray emission. As in the eROSITA data, its soft $\mathrm{X}$-ray emission is brighter toward the south. Figure 3 shows the RASS soft-band image of the relevant sky region. The effective survey exposure in the image varies from about $480 \mathrm{~s}$ at the eastern side of the remnant to about $474 \mathrm{~s}$ near to its central region and $380 \mathrm{~s}$ at its western side. ROSAT's scan direction imprint in that sky region is clearly visible in the image by the slightly inhomogeneous exposure, from approximately the southwestern to the northeastern direction. 


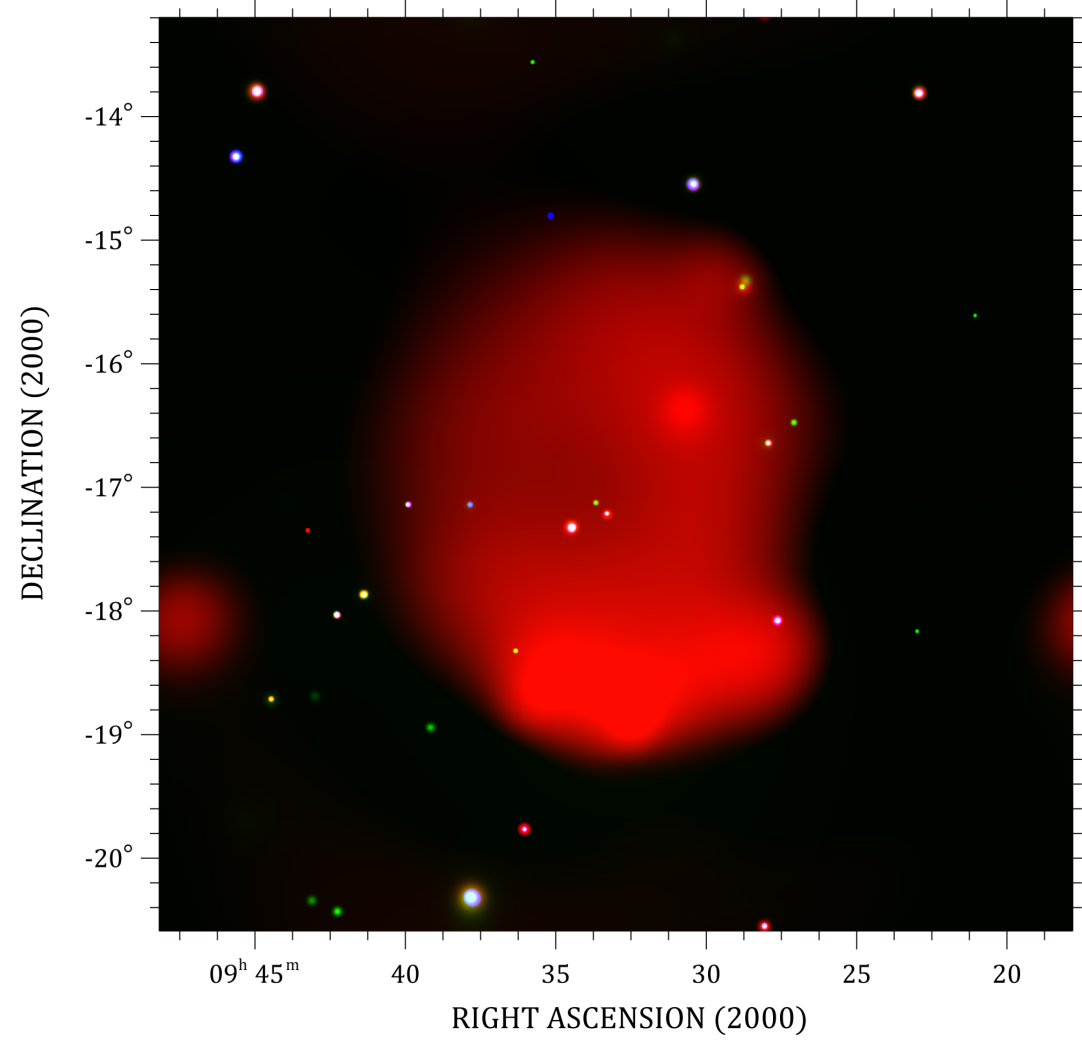

Fig. 2. Hoinga SNR as seen in the eROSITA all-sky survey eRASS1. Photons to produce this $7.5 \times 7^{\circ} .5$ image were colour coded according to their energy (red for energies $0.2-0.7 \mathrm{keV}$, green for $0.7-1.2 \mathrm{keV}$, blue for 1.2-2.4 keV). An adaptive kernel smoothing algorithm was applied to the images in each energy band.

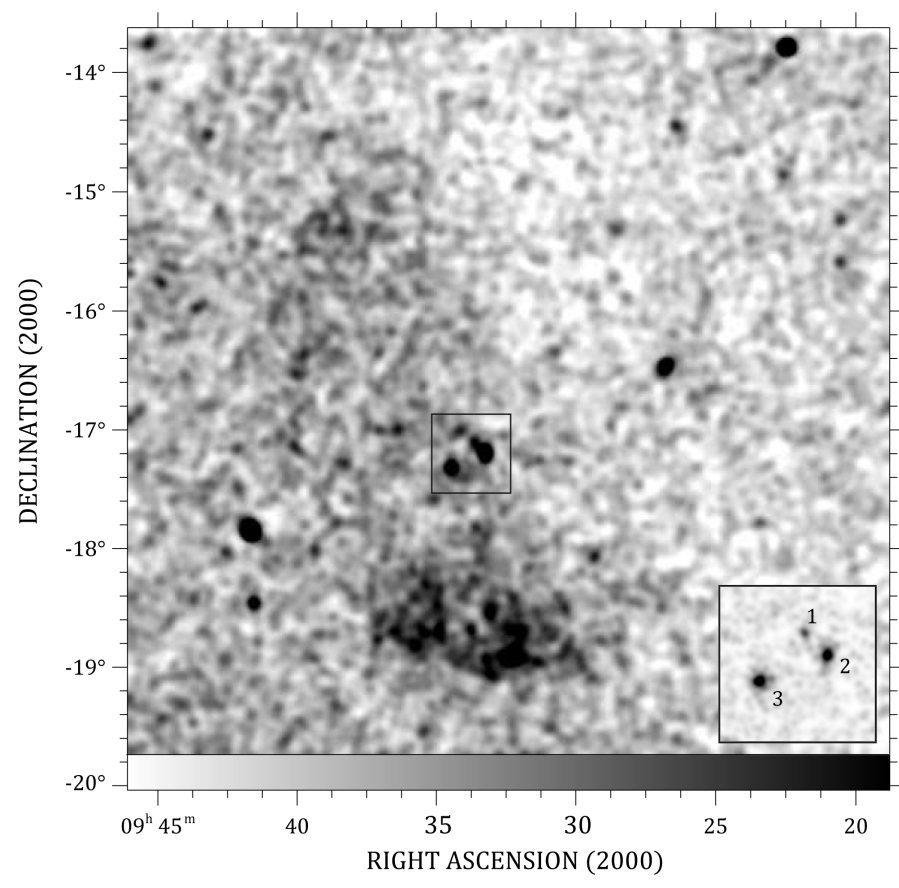

Fig. 3. Hoinga SNR as seen in the ROSAT all-sky survey. Photons to produce this image have been selected from within the $0.1-0.7 \mathrm{keV}$ energy band. A Gaussian smoothing filter with $\mathrm{x}, \mathrm{y}-\sigma=3$ image pixel was applied in order to enhance the visibility of the diffuse emission. The gray scale colors are distributed so that white corresponds to a pixel intensity value of 0.09 and black to $0.45 \mathrm{cts} \mathrm{pixel}^{-1}$. The image is vignetting and deadtime corrected though no exposure correction was applied. The inset in the lower right corner shows a $40^{\prime} \times 40^{\prime}$ zoom to the region of the X-ray sources located slightly to east of the remnant's geometrical centre.

\subsection{X-ray point sources within the Hoinga SNR}

In order to identify a possible compact remnant associated with Hoinga we applied a source detection to the ROSAT and eROSITA survey data. The point sources detected in both surveys along with their properties are summarised in Table 1 . The eROSITA $68 \%$ position uncertainty for point sources detected in the all-sky survey eRASS1 is 4.'5; for the ROSAT survey it is 13" (Voges et al. 1999). In Figs. 2 and 3, three point sources can be seen slightly to the east of the remnant geometrical centre, though rather centred with respect to the diffuse X-ray emission. While the positions of sources \#2 and \#3 remain unchanged within the errors in ROSAT and eROSITA data, source \#1 is found to have an offset towards the southeast of almost $20^{\prime \prime}$. For the purpose of a further source identification, we correlated the eROSITA positions with various radio and optical catalogues, for example NVSS (Condon et al. 1998) and Gaia DR2 (Gaia Collaboration 2018).

For the eROSITA sources \#2,\#3,\#5,\#6 we found a convincing positional match to a radio counterpart in the NVSS catalogue $^{3}$. For all sources, we find a close overlap with optical sources from the Gaia DR2 catalogue 4 . From the proper motion and parallax information for the potential counterparts, it seems likely that sources \#1,\#2,\#3,\#5, and \#6 are of extragalactic nature. In contrast, sources \#4, \#7, \#8, \#9, \#10, and \#11 appear to be likely of Galactic origin, in agreement with their brighter optical appearance.

Assuming the identification of the eROSITA source \#1 with an extragalactic optical source is correct, it seems more likely to us that the computed ROSAT RASS position of this faint

\footnotetext{
3 https://www.cv.nrao.edu/nvss

4 https://gea.esac.esa.int/archive
} 
Table 1. X-ray sources detected within the Hoinga SNR in eROSITA eRASS1 and ROSAT RASS data.

\begin{tabular}{cccc}
\hline \hline Source & $\begin{array}{c}\text { RA (J2000) } \\
\text { h:m:s }\end{array}$ & $\begin{array}{c}\text { Dec }(J 2000) \\
\text { d:m:s }\end{array}$ & $\begin{array}{c}\text { Obs. time } \\
\langle\text { MJD }\rangle\end{array}$ \\
\hline \multicolumn{4}{c}{ eROSITA eRASS1 } \\
\hline 1 & $09: 33: 41.096$ & $-17: 09: 18.932$ & 58987.40282 \\
2 & $09: 33: 18.088$ & $-17: 14: 41.741$ & 58987.31946 \\
3 & $09: 34: 30.071$ & $-17: 21: 21.224$ & 58987.65274 \\
4 & $09: 37: 57.489$ & $-17: 10: 14.453$ & 58988.23630 \\
5 & $09: 27: 29.469$ & $-18: 06: 20.653$ & 58986.48550 \\
6 & $09: 27: 50.522$ & $-16: 40: 01.672$ & 58985.98614 \\
7 & $09: 36: 25.814$ & $-18: 21: 05.829$ & 58988.40257 \\
8 & $09: 28: 45.469$ & $-15: 24: 10.805$ & 58985.73667 \\
9 & $09: 28: 38.097$ & $-15: 21: 08.361$ & 58985.73682 \\
10 & $09: 40: 02.199$ & $-17: 09: 55.614$ & 58988.65310 \\
11 & $09: 26: 58.572$ & $-16: 30: 06.584$ & 58985.73626 \\
\hline \multicolumn{5}{c}{ ROSAT RASS } \\
\hline 1 & $09: 33: 41.421$ & $-17: 08: 59.602$ & 48210.59365 \\
2 & $09: 33: 18.151$ & $-17: 14: 39.386$ & 48210.52687 \\
3 & $09: 34: 30.177$ & $-17: 21: 17.739$ & 48210.86065 \\
4 & $09: 37: 57.671$ & $-17: 10: 07.991$ & 48211.72799 \\
7 & $09: 36: 26.231$ & $-18: 21: 05.791$ & 48211.82793 \\
10 & $09: 40: 02.494$ & $-17: 09: 58.469$ & 48212.26215 \\
11 & $09: 26: 58.256$ & $-16: 30: 01.930$ & 48208.55845
\end{tabular}

Notes. The detection significance of the listed sources is $\geq 5 \sigma$. The position uncertainty of eROSITA point sources is 4 ". 5 ( $1 \sigma$ confidence). $\langle\mathrm{MJD}\rangle$ is the Modified Julian Date of the observation in eRASS1 and RASS, respectively. The numbering for the centrally located sources $\# 1$ - \#3 is reported in the lower-right inset of Fig. 3.

X-ray source has a larger uncertainty than the $13^{\prime \prime}$ found on average $(68 \%$ confidence) for ROSAT RASS sources (Voges et al. 1999). Assuming a real offset for source \#1 would imply a proper motion of $\sim 20^{\prime \prime} / 30 \mathrm{yr}$, which seems unlikely to us as we did not find a nearby bright star as optical counterpart. Indeed, of the $11 \mathrm{X}$-ray sources detected within the Hoinga SNR, none have an optical counterpart fainter than the twentieth magnitude in the Gaia $G$-band. Similarly, in the infrared band where the fainter object is found, $H \simeq 16$ and $\mathrm{W} 2(4.6 \mu \mathrm{m}) \simeq 14$ in $2 \mathrm{MASS}$ and WISE catalogues, respectively. We therefore conclude that all 11 $\mathrm{X}$-ray sources are either foreground or background objects which are not associated with Hoinga.

\subsection{Spectral analysis}

In order to properly correct the source spectrum and energy flux for contributions from the instrument- and sky-background, we analysed a sky field of about $8^{\circ} \times 8^{\circ}$ centred on the remnant. Hoinga's energy spectrum was extracted from the eROSITA eRASS1 data by selecting all events recorded within an elliptical region of semi-minor and major axis of $2^{\circ} .0$ and $2^{\circ} .35$, respectively. The elliptical region was centred at the position RA $=9: 32: 57.30$, Dec $=-16: 51: 41.00$ and tilted by 14.5 . SAOIMAGE DS9 (Joye \& Mandel 2003) was used for the definition of the event-selection regions. The background spectrum was extracted from a surrounding elliptical ring for which we chose the axes so that it did not include events from the remnant itself. The ring had a difference between its inner and outer region of 0.3 . Events from unrelated X-ray sources located within the

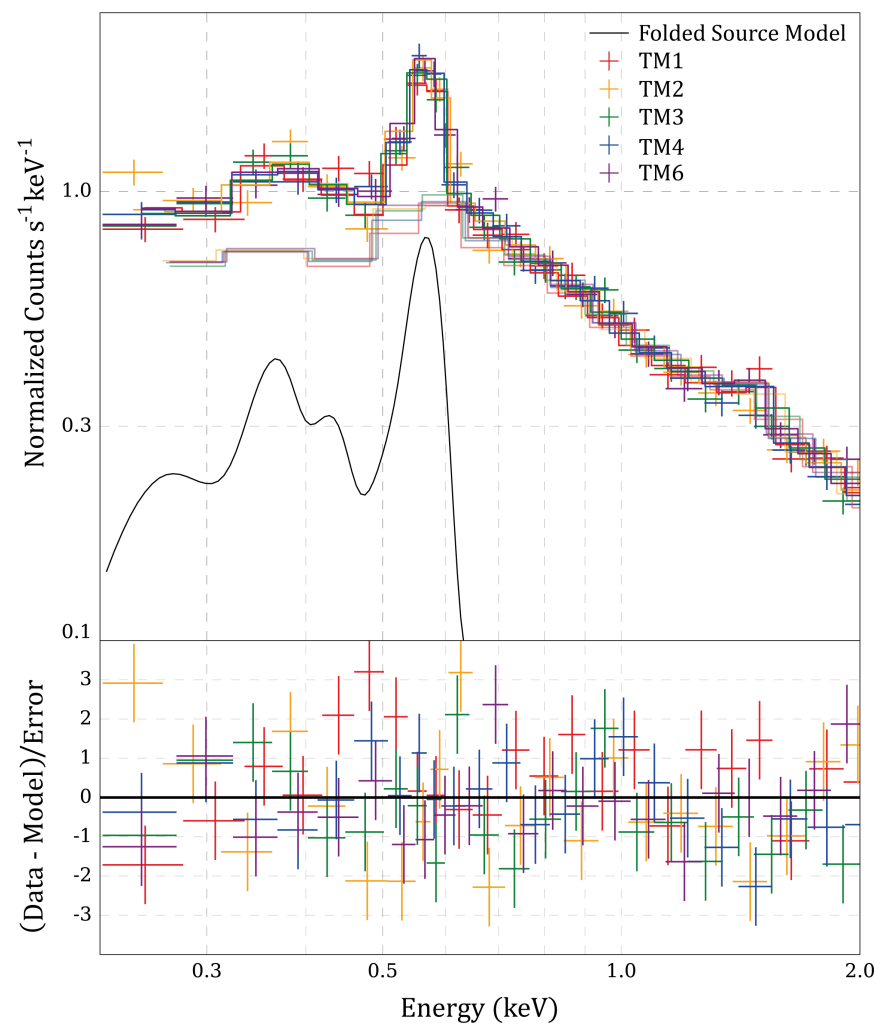

Fig. 4. Energy spectrum of the Hoinga SNR as observed with the eROSITA TM1, 2, 3, 4, and 6 telescope and detector units and simultaneously fitted to an absorbed APEC spectral model (upper panel). The spectra have been binned for visual clarity and plotting purposes. The signal-to-noise ratio in each bin is $15 \sigma$. The folded best-fit APEC spectral model is plotted as a solid black line. Fit residuals are shown in the lower panel.

source or background regions were excluded from the spectral analysis.

In total, the extracted spectra included 43910 and 28930 counts from the source and background regions, respectively, resulting in about 15000 net events. To model Hoinga's Xray spectrum, we used only events from the telescope units TM1, 2, 3, 4, and 6. Events from the two telescopes TM5 and TM7 were excluded from the spectral analysis as both units suffer light leaks related to the sun-satellite angle, making their soft-response calibration quite uncertain at that early stage of the mission. Model spectra were simultaneously fitted to Hoinga's source and background spectra. We used Xspec 12.10.1f (Dorman et al. 2003) and applied the C-statistics to the fits in which we modelled the source and background spectra independently. Of the fitted model spectra, the APEC spectrum from collisionally ionised diffuse gas (Foster et al. 2012) and the PSHOCK model (Plane-parallel SHOCK plasma model with non-equilibrium ionisation; Borkowski et al. 2001) were found to provide fits of equal goodness and with similar spectral parameters to the observed spectrum. We used the abundance table and the TBabs absorption model from Wilms et al. (2000). For the meaning of the fitted spectral parameters, we refer the reader to the $\mathrm{Xspec}$ manual ${ }^{5}$ and references therein.

Figure 4 depicts the best-fit APEC model. The model spectrum folded through the detector response is shown with a

\footnotetext{
5 https://heasarc.gsfc.nasa.gov/xanadu/xspec/ XspecManual.pdf
} 
Table 2. Best-fit parameters from the fit of APEC and PSHOCK models to the spectrum of Hoinga.

\begin{tabular}{ccc}
\hline \hline Parameter & APEC & PSHOCK \\
\hline$N_{\mathrm{H}}\left(10^{20} \mathrm{~cm}^{-2}\right)$ & $3.6_{-0.6}^{+0.7}$ & $3.6_{-1.0}^{+0.6}$ \\
$k T(\mathrm{keV})$ & $0.111_{-0.004}^{+0.004}$ & $0.108_{-0.008}^{+0.012}$ \\
$\tau\left(10^{11} \mathrm{~s} \mathrm{~cm}^{-3}\right)$ & $\ldots$ & $>1.1^{(a)}$ \\
Normalization & $0.17_{-0.03}^{+0.03}$ & $0.13_{-0.06}^{+0.06}$ \\
C Statistic / d.o.f. & $7625.1 / 7347$ & $7625.1 / 7346$ \\
\hline
\end{tabular}

Notes. Errors represent the $68 \%$ confidence range. ${ }^{(a)}$ The ionisation timescale $\tau$ is only weakly constrained by the fitted spectrum, which is why we only give a $95 \%$ lower limit.

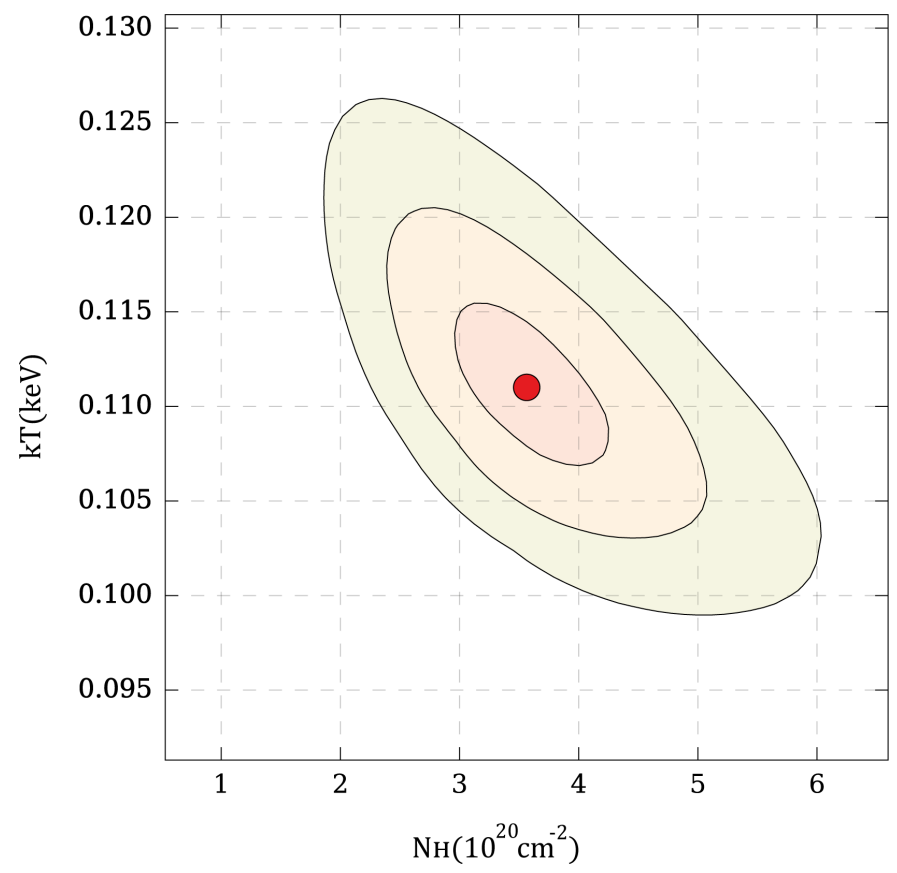

Fig. 5. Contour plot showing the relative parameter dependence of the fitted spectral parameters kT (temperature) vs. $N_{\mathrm{H}}$ (column absorption) for the APEC model fit to the energy spectrum of Hoinga. The three contours represent the $1 \sigma, 2 \sigma$, and $3 \sigma$ confidence levels for two parameters of interest. The small red dot marks the best-fit position.

black solid line. Table 2 lists the best-fit spectral parameters of both models. Due to the preliminary calibration status of the eROSITA instruments at the time of writing, we refrain from giving absolute energy fluxes as obtained from the best-fit models. The contour plot shown in Fig. 5 gives the parameter dependence of the temperature versus the column absorption for the APEC model.

\section{Radio observations and data analysis}

\subsection{The Murchison Widefield Array}

The Murchison Widefield Array (MWA; Tingay et al. 2013; Wayth et al. 2018) is a low-frequency radio telescope operating in Western Australia, and is a precursor to the low-frequency component of the Square Kilometre Array. The GaLactic and Extragalactic All-sky MWA (GLEAM; Wayth et al. 2015) survey observed the whole sky south of declination $(\mathrm{Dec})+30^{\circ}$ from 2013 to 2015 between 72 and $231 \mathrm{MHz}$. A major data release covering 24402 square degrees of extragalactic sky was published by Hurley-Walker et al. (2017), while individual studies have published smaller regions such as the Magellanic Clouds (For et al. 2018) and parts of the Galactic plane (HurleyWalker et al. 2019a). An important feature of this radio survey is its sensitivity to large-scale $\left(1^{\circ}-15^{\circ}\right)$ features, which has enabled studies of SNRs and HII regions across a wide range of sizes and the full range of frequencies, independent of resolution biases (see e.g. Hindson et al. 2016; Su et al. 2018; Hurley-Walker et al. 2019b).

Hoinga is visible in the public GLEAM images ${ }^{6}$ but is contaminated by the presence of hundreds of radio sources, the majority of which are likely unrelated radio galaxies (left panel of Fig. 6). To accurately measure the radio flux density of Hoinga, we reprocessed 13 two-minute observations spanning $103-231 \mathrm{MHz}$ from a drift scan centred at Dec $-13^{\circ}$ taken on 2014-03-04, with three or four observations in each $30.72-\mathrm{MHz}$ band, yielding integration times of $\approx 10 \mathrm{~min}$ per band. For each observation, we performed the following steps, in each case attenuating the brightness of modelled sources using the MWA primary beam model of Sokolowski et al. (2017):

- download the data from the All-Sky Virtual Observatory ${ }^{7}$ in standard measurement set format, averaged to $40 \mathrm{kHz}$ and $2 \mathrm{~s}$ frequency and time resolution;

- calculate a first-pass amplitude and phase calibration for each antenna using a sky model comprised of the bright nearby source Hydra A and the GLEAM catalogue, via the software CALIBRATE, an implementation of the MITCHCAL algorithm (Offringa et al. 2016);

- apply the derived calibration solutions;

- use the PEEL software to remove Hydra A from the visibilities, with a solution interval of $4 \mathrm{~s}$;

- directly subtract the GLEAM sources from the visibilities using SUBTRMODEL;

- use the widefield radio imaging package WSCLEAN (Offringa et al. 2014) to image the data using natural weighting and multi-scale multi-frequency synthesis over the full 30.72-MHz band down to a threshold of three times the local image noise, and then clean the data down to the local image noise in regions found to contain brightness.

The ionosphere was found to be in a relatively quiescent state, with minor $(\approx \operatorname{arcsec})$ position shifts imparted to the radio sources; the images were corrected using FITS_WARP (Hurley-Walker \& Hancock 2018). For each 30.72-MHz band, the primary-beam-corrected images were then mosaicked using SWARP (Bertin et al. 2002). The resulting image is shown in the right panel of Fig. 6. Hoinga is visible as a pair of arcs of width $\approx 1^{\circ}, 5^{\circ}$ apart from one another. The local diffuse Galactic synchrotron is also visible as a fainter series of filaments with a similar colour (i.e. spectral index).

We used the software POLY_FLUX (Hurley-Walker et al. 2019c) to measure the total flux densities of Hoinga in each band, estimating and subtracting a mean background level. As the selection of the boundaries of the SNR is somewhat subjective, we used the tool ten times and recorded the average result. The results are shown in Table 3 . The uncertainties are estimated at $20 \%$, dominated by the difficulty in selecting the true bounds of the SNR and calculating the true background level of the Galactic cirrus.

\footnotetext{
6 http://gleam-vo.icrar.org/gleam_postage/q/form 7 https://asvo.mwatelescope.org/
} 

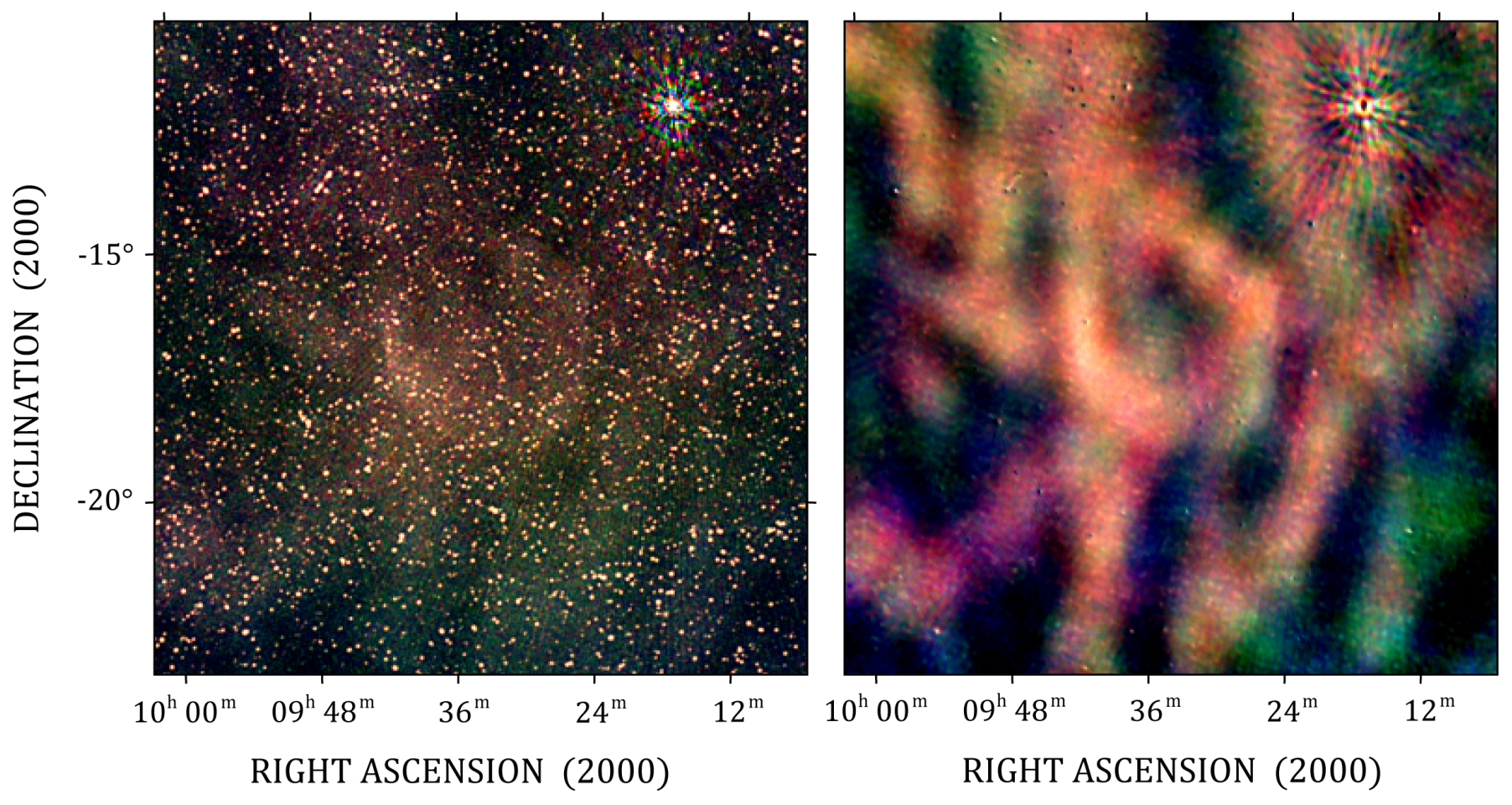

Fig. 6. $\sim 10^{\circ} \times 10^{\circ}$ of the region surrounding Hoinga as seen by GLEAM at 103-134 MHz (R), 139-170 MHz (G), and 170-200 MHz (B). Left panel: image from the data release of Hurley-Walker et al. (2017), and right panel: region after reprocessing to subtract sources and highlight large-scale structure (see Sect. 3.1). Hoinga is visible as an ellipse in the centre of the image; steep-spectrum Galactic cirrus becomes a strong contaminant at these low frequencies and is visible as large-scale filaments around the remnant. The bright source in the northwest is Hydra A.

Table 3. Integrated flux densities of Hoinga measured from the radio data described in Sect. 3.

\begin{tabular}{cccc}
\hline \hline Survey & $\begin{array}{c}\text { Frequency } \\
(\mathrm{MHz})\end{array}$ & $\begin{array}{c}\text { Resolution } \\
\left({ }^{\prime}\right)\end{array}$ & $\begin{array}{c}\text { Flux density } \\
(\mathrm{Jy})\end{array}$ \\
\hline GLEAM & 118 & $7.8 \times 6.6$ & $115 \pm 23$ \\
GLEAM & 154 & $6.0 \times 5.0$ & $100 \pm 20$ \\
GLEAM & 185 & $5.0 \times 4.2$ & $90 \pm 18$ \\
GLEAM & 215 & $4.2 \times 3.6$ & $80 \pm 16$ \\
Haslam & 408 & 51 & $60 \pm 10$ \\
CHIPASS & 1400 & 14.4 & $19.7 \pm 1.0$ \\
SPASS & 2300 & 8.9 & $15.2 \pm 0.3$ \\
\hline
\end{tabular}

Notes. Measurements were made on images where contaminating sources and background had been removed using the software POLY_FLUX.

\subsection{Haslam}

The all-sky 408-MHz 'Haslam' survey was performed with the Green Bank and Parkes Radio telescopes and remains the lowestfrequency total-power measurement of the full sky (Haslam et al. 1982). The Hoinga SNR is visible in the Haslam images (Fig. 7) but the scanning pattern of Parkes is visible as a series of vertical lines of varying brightness throughout the image. As this is a total power measurement, the largest scale Galactic cirrus features are much brighter than Hoinga, leading to a large increase in brightness between the east and west parts of the image. The images are also invisibly contaminated by the same radio sources resolved in the GLEAM data (Sect. 3.1). To mitigate these issues, we used the following steps:

- model and subtract the GLEAM extragalactic catalogue for this region, extrapolating the source spectra to $408 \mathrm{MHz}$, either via their spectral index $\alpha$ as measured by GLEAM or for the fainter sources, by an assumed value of -0.75 ;

- determine the average brightness profile over the lower portion of the image (south of Hoinga) as a function of right ascension, and subtract this profile from the full image.

This resulted in the right-hand panel of Fig. 7, where the artefacts and contaminating sources have largely been removed. Similarly to the GLEAM data, we ran POLY_FLUX and found that the uncertainty on the final results was dominated by the difficulty in subtracting the background, which still has large scan artefacts. We therefore conservatively estimate the error at $20 \%$.

We also attempted to use the 'de-striped' 'de-sourced' version of the Haslam image produced by Remazeilles et al. (2015), but Hoinga was invisible in this version, possibly because it has similar angular scale to the scanning artefacts, and so was removed by the clean-up algorithms employed.

\subsection{CHIPASS}

The continuum map of the HI Parkes All-Sky Survey (CHIPASS; Calabretta et al. 2014) maps the radio sky at $1.4 \mathrm{GHz}$ south of Dec $+25^{\circ}$. We downloaded the data ${ }^{8}$, and cropped and regridded it to match the MWA mosaics (left panel of Fig. 8). We selected sources within $15^{\circ}$ of Hoinga from the NRAO VLA Sky Survey (NVSS; Condon et al. 1998), convolved them to match the CHIPASS resolution, and produced an output FITS image in the same sky frame as the regridded CHIPASS data. We subtracted the NVSS model from the CHIPASS image, producing the right panel of Fig. 8. We used POLY_FLUX to measure the

\footnotetext{
8 https://www.atnf.csiro.au/people/mcalabre/CHIPASS/ index.html
} 

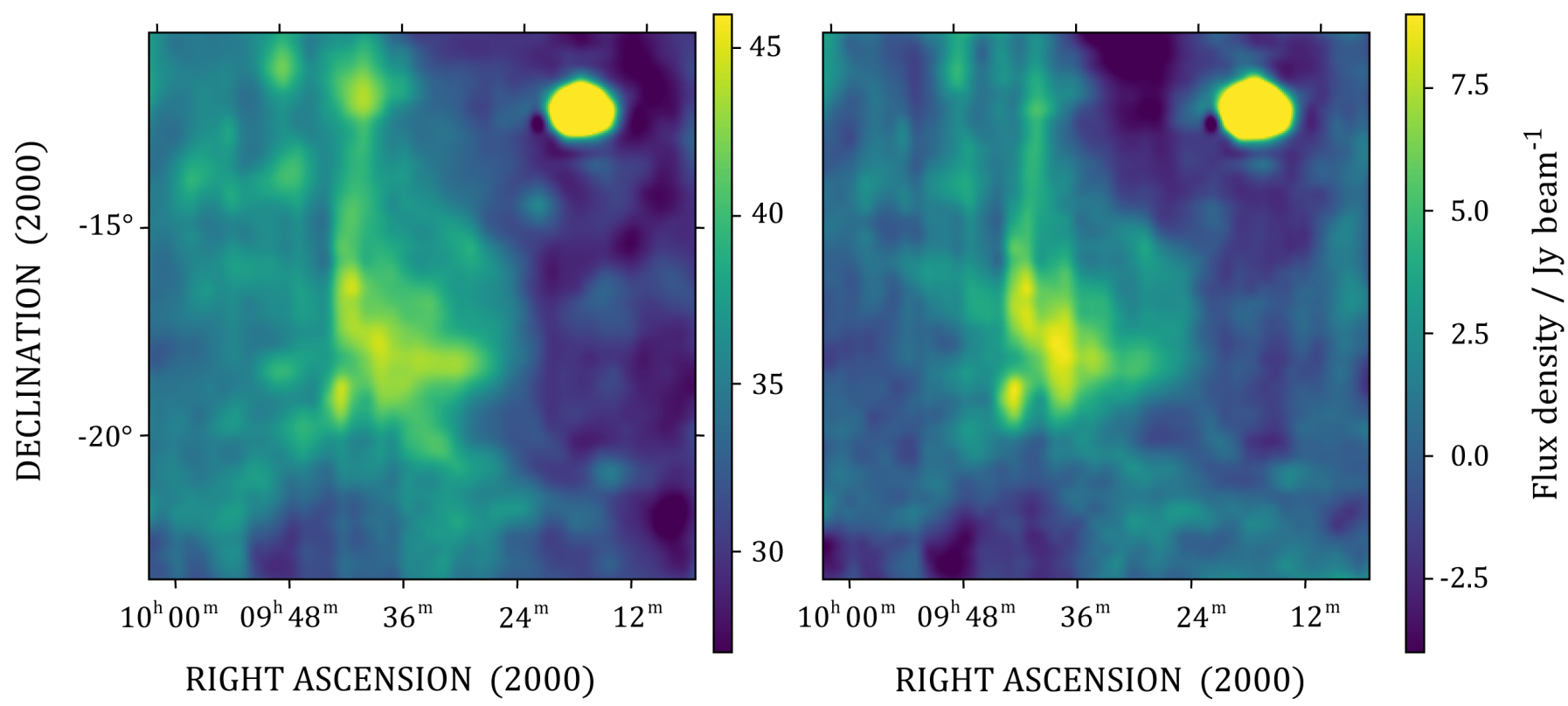

Fig. 7. $100 \mathrm{deg}^{2}$ of the region surrounding the Hoinga SNR as seen at $408 \mathrm{MHz}$ by the survey by Haslam et al. (1982), after conversion from K to $\mathrm{Jy} \mathrm{beam}^{-1}$. Left panel: original image, and right panel: image after source-subtraction and backgrounding, discussed in Sect. 3.2. Hoinga is visible as an ellipse in the centre of the image, while Galactic cirrus and scan line artefacts from the Parkes observing strategy dominate the surroundings. The bright source in the northwest is Hydra A, and subtraction of this source has not been performed.
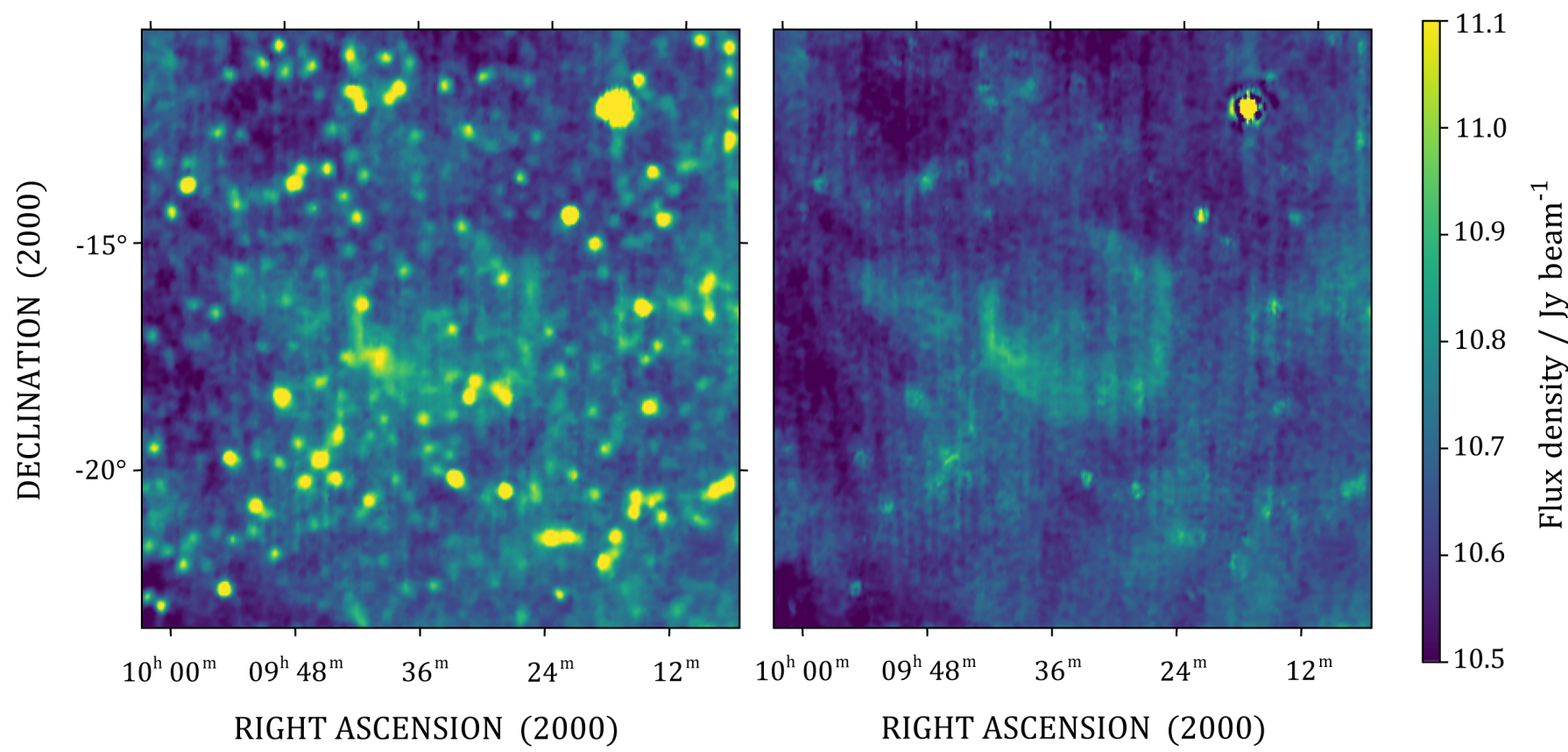

Fig. 8. $100 \mathrm{deg}^{2}$ of the region surrounding Hoinga as seen at $1.4 \mathrm{GHz}$ by CHIPASS, after conversion from $\mathrm{K}$ to Jy beam ${ }^{-1}$. Left panel: original image, and right panel: image after source subtraction, discussed in Sect. 3.3. Hoinga is clearly visible as a crescent-moon in the centre of the image, while Galactic cirrus and residuals around poorly subtracted diffuse sources are visible in the surroundings. The bright source in the northwest is Hydra A. Faint scan lines are visible from the Parkes observing strategy.

flux density of Hoinga, shown in Table 3. The errors are dominated by the selection of the region for subtraction, and after repeated measurements, we estimate this at about $5 \%$, which is $1 \mathrm{Jy}$.

\subsection{S-PASS}

The S-Band Polarization All Sky Survey (SPASS ; Carretti et al. 2019) is a survey of polarized radio emission over the southern sky at Dec $<-1^{\circ}$ using the Parkes radio telescope at $2.3 \mathrm{GHz}$. Unlike for CHIPASS (Sect. 3.3) there is no independent catalogue of extragalactic radio sources at $2.3 \mathrm{GHz}$. Meyers et al. (2017) derived a catalogue of radio sources from a version of the S-PASS images where the large-scale emission had been filtered out, with slightly worse resolution $(10.75)$ than the published images (8'.9). This catalogue is not as sensitive as and is more confused than NVSS yielding a source density equal to $3 \%$ that of NVSS. 

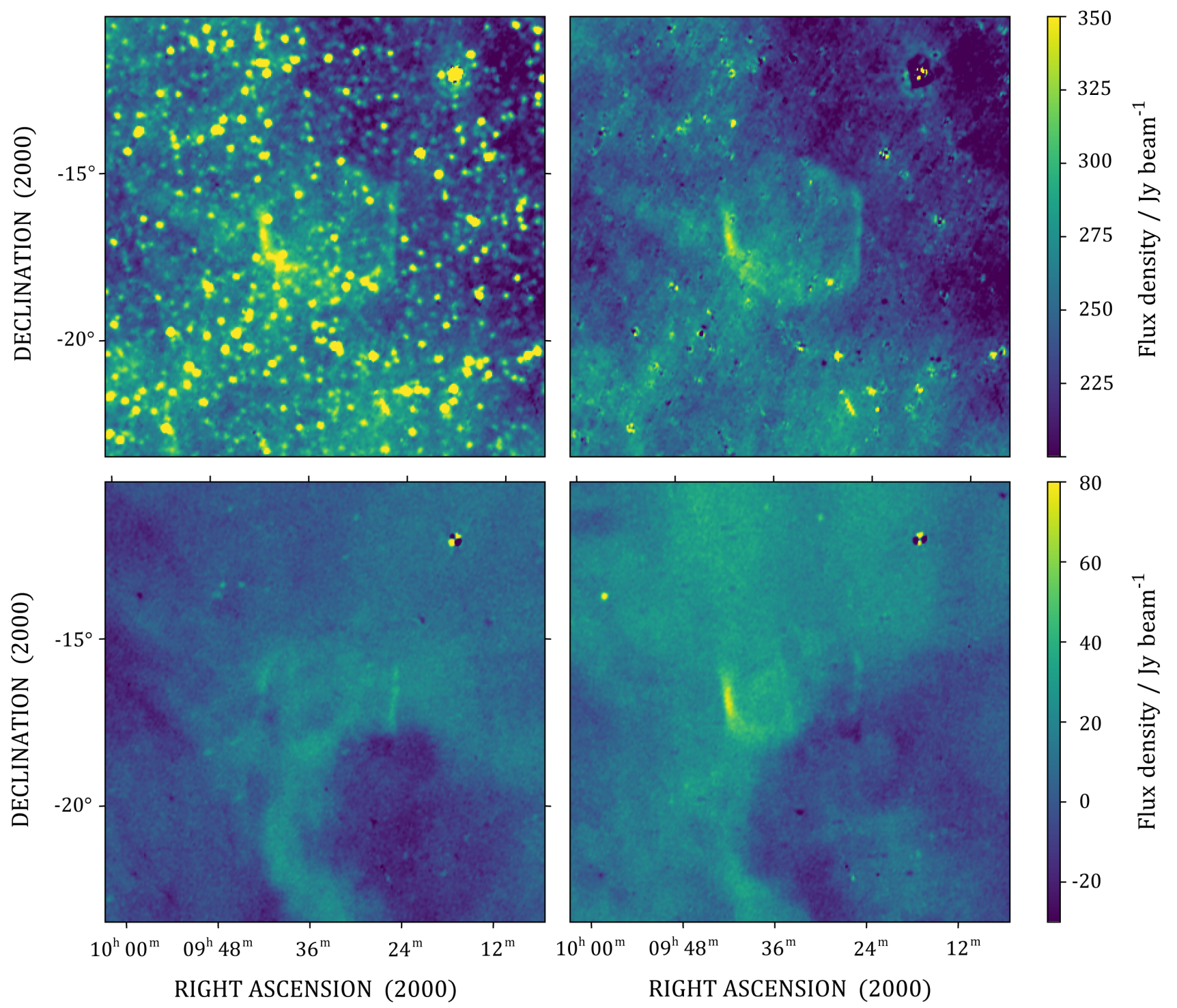

Fig. 9. $100 \mathrm{deg}^{2}$ of the region surrounding Hoinga as seen at $2.3 \mathrm{GHz}$ by SPASS. Top-left panel: Stokes I image, and top-right panel: image after source subtraction, discussed in Sect. 3.4. Hoinga is clearly visible as a filled ellipse in the centre of the image, while Galactic cirrus and residuals around poorly subtracted diffuse sources are visible in the surroundings. Bottom left and bottom right panels: Stokes $Q$ and $U$ images, respectively.

We therefore use NVSS to create the local model of sources to subtract. To obtain spectral indices for each source, we use the catalogue produced by de Gasperin et al. (2018); for sources without a listed spectral index, we use the median local value of $\alpha=-0.75$. Subtracting this model from the S-PASS data results in the right-hand panel of Fig. 9. Running POLY_FLUX repeatedly we find more consistent results than for CHIPASS; the uncertainty is most likely dominated by the less clean source subtraction. The residual RMS after source subtraction in a given beam is $\approx 20 \mathrm{mJy}_{\text {beam }}{ }^{-1}$; Hoinga subtends 256 SPASS beams; the error is therefore estimated as $0.32 \mathrm{Jy}$.

As S-PASS is a polarisation survey, we can also examine the Stokes $Q$ and $U$ images of the region, which indicate the degree of linear polarisation at angles of $\pm 90^{\circ}$ and $\pm 45^{\circ}$, respectively. Figure 9 shows that the brightest parts of the shell (left and right 'limbs') show clear linear polarisation, which is what would be expected from a middle-aged SNR shell with a large shock compression ratio. These also correspond to flatter parts of the SNR shell, perhaps indicating a local increase in gas density.

\section{Constraints on ${ }^{44} \mathrm{Ti}$ emission from INTEGRAL}

Explosive nucleosynthesis in $\mathrm{SNe}$ is considered the main driver of Galactic, chemical evolution. Its imprints can be readily investigated by observing the $\gamma$-rays emitted in the decay from freshly synthesized, radioactive nuclei. With a half-life of $58.9 \mathrm{yr}$, the abundantly produced ${ }^{44} \mathrm{Ti}$ is an ideal candidate with which to study nucleosynthesis imprinting in young SNRs.

In core collapse supernovae (ccSN) ${ }^{44} \mathrm{Ti}$ is mainly produced during the $\alpha$-rich freeze-out (Woosley et al. 1973) deep in the central region, where the nucleosynthesis yields are strongly dependent on the thermodynamic conditions (Magkotsios et al. 2010; Hermansen et al. 2020). While models of ccSN fail to robustly produce explosions in a wide stellar mass range so far, it appears safe to assume that asymmetries are required to drive successful explosions. Depending on the applied, simplified explosion scheme, the predicted ${ }^{44} \mathrm{Ti}$ ejecta yield can vary in the range $10^{-5}-10^{-4} M_{\odot}$, depending also on the initial mass of the exploding star (Timmes et al. 1996; Wanajo et al. 2018; Limongi \& Chieffi 2018). 
In contrast, thermonuclear SNe (type Ia) show a larger diversity in the predicted ${ }^{44} \mathrm{Ti}$ ejecta masses. Multiple scenarios leading to the disruption of a white dwarf star are considered viable, as the progenitors of these explosions have not yet been unambiguously identified. For the standard model, involving a centrally ignited Chandrasekhar-mass white dwarf star, ${ }^{44} \mathrm{Ti}$ ejecta masses range between $10^{-6}$ and $10^{-5} M_{\odot}$ (Maeda et al. 2010; Seitenzahl et al. 2013; Fink et al. 2014). However, in the double-detonation scenario, ejecta masses of $10^{-3}-10^{-2} M_{\odot}$ are possible (Fink et al. 2010; Woosley \& Kasen 2011; Moll \& Woosley 2013), where some exotic models even predict ${ }^{44} \mathrm{Ti}$ masses of up to $0.1 M_{\odot}$ (Perets et al. 2010; Waldman et al. 2011).

Evidence for the production of ${ }^{44} \mathrm{Ti}$ can be obtained by measuring the decay radiation in the decay chain of ${ }^{44} \mathrm{Ti} \rightarrow$ ${ }^{44} \mathrm{Sc} \rightarrow{ }^{44} \mathrm{Ca}$. The dominant decay lines are emitted at 68 and $78 \mathrm{keV}$ during the ${ }^{44} \mathrm{Ti}$ decay with a half life of $58.9 \mathrm{yr}$ (Ahmad et al. 2006) and at $1157 \mathrm{keV}$ in the subsequent ${ }^{44} \mathrm{Sc}$ decay with a half life of $4 \mathrm{~h}$ (Audi et al. 2003). Photons are emitted with a probability (branching ratio) of 93.0, 96.4, and 99.9\% per decay, respectively (Chen et al. 2011)

Here, the spectrometer SPI (Vedrenne et al. 2003) on INTEGRAL (Winkler et al. 2003) is used to search for the decay radiation in both subsequent decay steps in the Hoinga SNR. We use the spimodfit analysis tool (Strong et al. 2005; Halloin 2009) to extract the spectrum in the relevant energy ranges 50-100 and $1100-1200 \mathrm{keV}$ from the raw SPI data. The spectrum is extracted assuming an extended source of emission modelled by a circular region of 2.2 radius with a constant surface brightness. A detailed description of SPI analysis and robust background modelling can be found in Diehl et al. (2018), Siegert et al. (2019) and Weinberger et al. (2020).

The extracted spectrum is modelled with a general continuum and a variable number of Gaussian-shaped decay lines given by

$\operatorname{LS}\left(E ; E_{0}, F_{0}, \sigma\right)=\frac{F_{0}}{\sqrt{2 \pi} \sigma} \cdot \exp \left(\frac{\left(E-E_{0}\right)^{2}}{2 \sigma^{2}}\right)+A_{0} \cdot\left(\frac{E}{E_{C}}\right)^{\alpha}$,

where $F_{0}$ is the measured line flux, $E_{0}$ is the energy of the Doppler-shifted line centroid, and $\sigma$ is the line width. As we expect a low signal-to-noise ratio for the decay lines, we search for a combined signal in all lines simultaneously, that is we assume that the branching ratio corrected fluxes, Doppler shifts, and broadening are identical in all lines. Due to the presence of a complex of strong background lines between 50 and $65 \mathrm{keV}$ induced by germanium, we excluded the $68 \mathrm{keV}$ line in the analysis.

We find no significant flux excess in the vicinity of the 78 or $1157 \mathrm{keV}$ line or in the combined line analysis. As the broadening of the 78 or $1157 \mathrm{keV}$ lines is related to the expansion velocity of the ${ }^{44} \mathrm{Ti}$-containing ejecta and determines the size of the selected background region, we deduce a $3 \sigma$ upper flux limit of $9.2 \times 10^{-5} \mathrm{ph} \mathrm{cm}^{-2} \mathrm{~s}^{-1}$ by assuming an expansion velocity of $4000 \mathrm{~km} \mathrm{~s}^{-1}$ (Nagataki et al. 1998; Diehl et al. 2015). This expansion velocity translates into a line broadening of $\approx 2 \mathrm{keV} \mathrm{FWHM}$ at $78 \mathrm{keV}$ and $\approx 20 \mathrm{keV} \mathrm{FWHM}$ at $1157 \mathrm{keV}$, respectively.

\section{Summary and discussion}

Using data from the first SRG/eROSITA observatory all-sky survey we discovered one of the largest SNRs in the sky. Despite $95 \%$ of SNR discoveries being made at radio wavelengths, and its clear existence in multiple radio surveys, we conclude that Hoinga was missed by previous searches for several reasons.

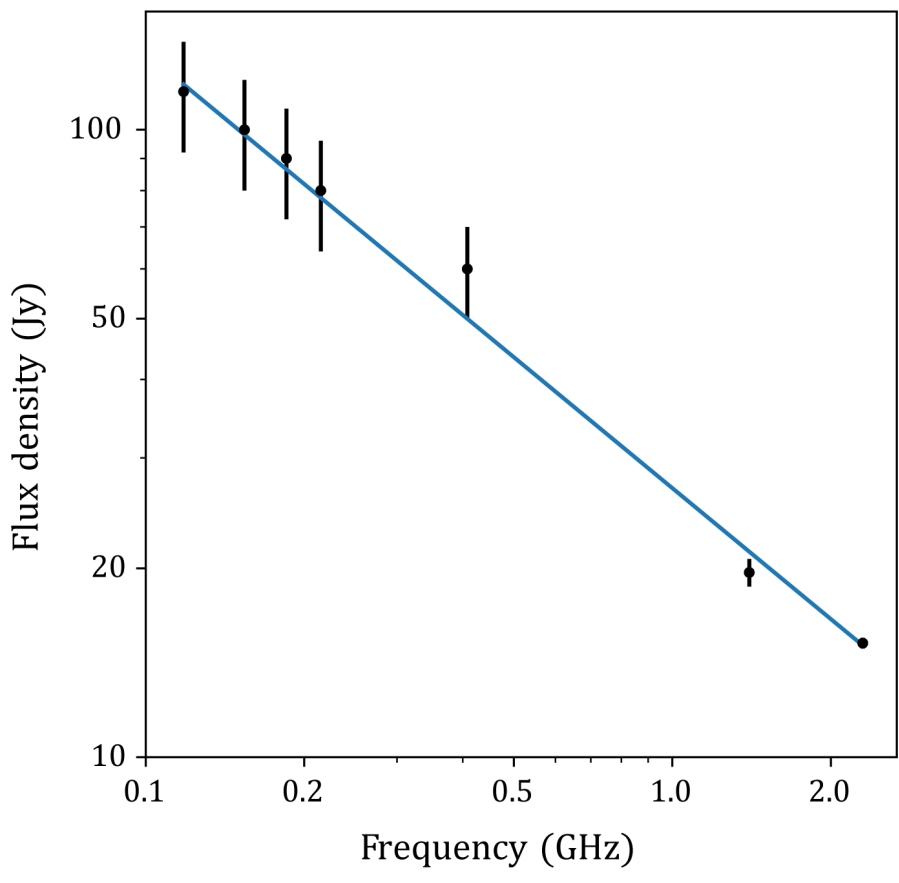

Fig. 10. Radio SED of the total flux density of Hoinga as measured by the surveys discussed in Sect. 3. Black points show the data from Table 3; the blue line shows a least-squares weighted fit to the data, yielding $S_{1 \mathrm{GHz}}=26.85 \pm 0.03 \mathrm{Jy}$ and $\alpha=-0.69 \pm 0.08$ for $S_{v} \propto \nu^{\alpha}$.

Firstly, its location at high Galactic latitudes; most radio searches have focused on low latitudes, where the density of SNRs is expected to be highest. Another reason for not noticing it in previous X-ray and radio surveys is its total flux density. Although it is large, its surface brightness is relatively low. As it has very little fine-scale structure, it also does not appear at all in most interferometric maps. In single-dish radio images, it is visibly contaminated by about 100 extragalactic radio sources, with many more below the sensitivity and confusion limits, meaning that its diffuse radio emission remained uncovered. Hoinga is nearly the largest SNR ever detected at radio wavelengths, subtending $\approx 275^{\prime} \times 265^{\prime}$, and comparable in size to the largest detected object, G 65.3+5.7: it was therefore outside the bounds of what was expected and was therefore not visually detected. Finally, its similar angular scale and structure to the diffuse Galactic synchrotron makes it less obvious than smaller and brighter sources.

The clear shell structure, particularly evident in Figs. 1 and 8, indicates it is likely to be a classic shell-type SNR that is not centrally powered, and its highly circular nature indicates that it is expanding into a region of relatively uniform density. Figure 10 shows the radio flux densities plotted as a function of frequency, with a fitted spectral index of $\alpha=-0.69 \pm 0.08$, for $S_{\nu} \propto$ $v^{\alpha}$. This radio spectral energy distribution indicates that nonthermal synchrotron emission dominates the radio spectrum, again consistent with a shell-type SNR.

A distance to the SNR would enable transformation of our measurements into physical properties. Dubner \& Giacani (2015) discuss the challenge of estimating the distance of radio-detected SNRs; a method that does not rely on additional observations is to search for nearby neutron stars that appear as pulsars and may have formed at the same time as the SNR, and using their dispersion measure in combination with electron density models of the Galaxy to determine their distance. 
We used the Australia Telescope National Facility pulsar catalogue v1.59 (Manchester et al. 2005) ${ }^{9}$ to search for known radio pulsars within $20^{\circ}$ of Hoinga's geometrical centre, but found none with attributes that would indicate a clear association. From the group of pulsars located in the region of interest we excluded possible matches on the basis of:

- period $P<10 \mathrm{~ms}$, indicating a recycled origin;

- characteristic ages $\left(\frac{P}{2 \dot{P}}\right)>45 \mathrm{Myr}$, which would be extremely inconsistent with a SNR age of $<0.5 \mathrm{Myr}$;

- measured proper motion inconsistent with having a common centre of origin;

- measured dispersion measure inconsistent with a nearby location.

This avenue is therefore unpromising, but because the coverage of pulsar surveys is denser at low Galactic latitude, a pulsar could have been missed by existing observations, and follow-up observations within the SNR shell may yet reveal a counterpart. Assuming a distance of $\sim 500 \mathrm{pc}$, a remnant NS with a transversal speed of the order of $1000 \mathrm{~km} \mathrm{~s}^{-1}$ would have by now reached the SN shell if the explosion happened $\sim 17000 \mathrm{yr}$ ago. This speed is not unrealistic, albeit at the far side of the velocity distribution (see e.g. Cordes et al. 1993; Chatterjee et al. 2005; Becker 2009). We will investigate this possibility in future work.

In the absence of a measured distance, we can use the morphological and brightness properties of the SNR to infer limits on the physical characteristics. Studies of the Magellanic Clouds and other Local Group galaxies show that SNR 1.4-GHz luminosities typically have values in the range $5 \times 10^{14}<L_{1.4 \mathrm{GHz}}<$ $10^{17} \mathrm{~W} \mathrm{~Hz}^{-1}$ (e.g. Case \& Bhattacharya 1998). Assuming that Hoinga is more luminous than $5 \times 10^{14} \mathrm{~W} \mathrm{~Hz}^{-1}$, we can obtain a limit on its distance from Earth by $\sqrt{\frac{L_{1.4 \mathrm{GHz}}}{4 \pi S_{1.4 \mathrm{GHz}}}}$, i.e. $D>450 \mathrm{pc}$. Additionally, radio SNRs do not typically have diameters greater than $100 \mathrm{pc}$ (Badenes et al. 2010). If we assume that Hoinga has a diameter $<100 \mathrm{pc}$, by geometry its distance from Earth must be $D<1.2 \mathrm{kpc}$. This also gives rise to a luminosity limit of $L_{1.4 \mathrm{GHz}}<1.3 \times 10^{16} \mathrm{~W} \mathrm{~Hz}^{-1}$, which puts Hoinga on the lower end of the SNR luminosity distribution. We note that other high-latitude SNRs have also been found to have unusually low brightness compared to those at low latitudes; see e.g. G181.1+9.5 (Kothes et al. 2017) and G 0.1-9.7 (Hurley-Walker et al. 2019b).

If we compare the remnant with other nearby SNRs such as the Vela SNR, which is also known to have an extent of 8.8 and a thermal X-ray spectrum with gas temperatures in the range of $0.2-0.7 \mathrm{keV}$, a simple scaling law puts Hoinga at twice the distance of the Vela SNR, which is about $500 \mathrm{pc}$. The column absorption through the Galaxy into the direction of Hoinga is $6 \times 10^{20} \mathrm{~cm}^{-2}$ (Dickey \& Lockman 1990). The values found from our X-ray spectral fits are of the order of $N_{\mathrm{H}}=3.6_{-0.6}^{+0.7} \times 10^{20} \mathrm{~cm}^{-2}$ which gives another indication for Hoinga being a nearby SNR.

If we assume that the column density derived in Sect. 2.4 is representative along the entire line of sight, we can derive a range of local ISM densities by dividing by the distance limits. For a column density of $N_{\mathrm{H}}=6 \times 10^{20} \mathrm{~cm}^{-2}$, and distances of $0.45-1.2 \mathrm{kpc}$, the resulting local density $n_{\mathrm{H}}=0.42-0.16 \mathrm{~cm}^{-3}$. Inputting these into the SNR evolutionary model calculator provided by Leahy \& Williams (2017), with otherwise standard model and input values, we calculate the range of possible ages as $21-150$ kyr. However, the morphology of the SNR suggests a

\footnotetext{
9 atnf.csiro.au/research/pulsar/psrcat/
}

much lower age, and therefore we suggest the SNR is likely to be at the closer, younger, and higher $n_{\mathrm{H}}$ ends of the allowable ranges.

Taking into account the fact that no pulsar has been associated with the object so far, it is highly possible that Hoinga is the remnant of a type Ia SN. This would also be consistent with the high latitude of the SNR, as the massive star progenitors of core-collapse $\mathrm{SNe}$ are expected to be more concentrated in the Galactic plane (Taylor et al. 1993; Cordes \& Lazio 2002; Faucher-Giguere \& Kaspi 2006).

eROSITA will perform a total of eight all-sky surveys. With further surveys completed, more data from the Hoinga remnant will become available in the next few years. This will allow us to study the remnants fine structure and spectral properties in more detail, hopefully allowing us to further constrain its distance, age, chemical composition, and SN type. The findings of Hoinga represent a highlight of the beginning of a wider program setup by the authors WB and NHW as part of an eROSITAAustralian-based joint-venture collaboration defined to explore the X-ray-radio-sky in order to uncover further exciting surprises in the SNR sphere.

Acknowledgements. We thank Bernd Aschenbach and Nicholas Pingel for fruitful discussions and the anonymous referee for valuable comments. eROSITA is the primary instrument aboard SRG, a joint Russian-German science mission supported by the Russian Space Agency (Roskosmos), in the interests of the Russian Academy of Sciences represented by its Space Research Institute (IKI), and the Deutsches Zentrum für Luft- und Raumfahrt (DLR). The SRG spacecraft was built by Lavochkin Association (NPOL) and its subcontractors, and is operated by NPOL with support from IKI and the Max Planck Institute for Extraterrestrial Physics (MPE). The development and construction of the eROSITA X-ray instrument was led by MPE, with contributions from the Dr. Karl Remeis Observatory Bamberg \& ECAP (FAU Erlangen-Nürnberg), the University of Hamburg Observatory, the Leibniz Institute for Astrophysics Potsdam (AIP), and the Institute for Astronomy and Astrophysics of the University of Tübingen, with the support of DLR and the Max Planck Society. The Argelander Institute for Astronomy of the University of Bonn and the Ludwig Maximilians Universität Munich also participated in the science preparation for eROSITA. The eROSITA data shown here were processed using the eSASS/NRTA software system developed by the German eROSITA consortium. N.H.W. is supported by an Australian Research Council Future Fellowship (project number FT190100231) funded by the Australian Government. This scientific work makes use of the Murchison Radio-astronomy Observatory, operated by CSIRO. We acknowledge the Wajarri Yamatji people as the traditional owners of the Observatory site. Support for the operation of the MWA is provided by the Australian Government (NCRIS), under a contract to Curtin University administered by Astronomy Australia Limited. Establishment of the Murchison Radio-astronomy Observatory and the Pawsey Supercomputing Centre are initiatives of the Australian Government, with support from the Government of Western Australia and the Science and Industry Endowment Fund. We acknowledge the Pawsey Supercomputing Centre which is supported by the Western Australian and Australian Governments. Access to Pawsey Data Storage Services is governed by a Data Storage and Management Policy (DSMP). ASVO has received funding from the Australian Commonwealth Government through the National eResearch Collaboration Tools and Resources (NeCTAR) Project, the Australian National Data Service (ANDS), and the National Collaborative Research Infrastructure Strategy. This research has made use of NASA's Astrophysics Data System Bibliographic Services. M.G.F.M. acknowledges support by the International Max-Planck Research School on Astrophysics at the Ludwig-Maximilians University, IMPRS. This work has made use of data from the European Space Agency (ESA) mission Gaia (https: //www. cosmos. esa.int/gaia), processed by the Gaia Data Processing and Analysis Consortium (DPAC, https://www.cosmos.esa.int/web/ gaia/dpac/consortium). Funding for the DPAC has been provided by national institutions, in particular the institutions participating in the Gaia Multilateral Agreement. The following 3rd-party software was used in this work: AOFLAGGER and COTTER (Offringa et al. 2015); WSCLEAN (Offringa et al. 2014; Offringa \& Smirnov 2017); AEGEAN (Hancock et al. 2018); MIRIAD (Sault et al. 1995); TOPCAT (Taylor 2005) NUMPY v1.11.3 (Dubois et al. 1996; Harris et al. 2020); ASTROPY v2.0.6 (Astropy Collaboration 2013); ScIPY v0.17.0 (Oliphant 2007), MATPLOTLIB v1.5.3 (Hunter 2007). The manuscript was prepared on the web-based LATEX editor, Overleaf. 


\section{References}

Adams, S. M., Kochanek, C. S., Gerke, J. R., Stanek, K. Z., \& Dai, X. 2017 MNRAS, 468, 4968

Ahmad, I., Greene, J. P., Moore, E. F., et al. 2006, Phys. Rev. C, 74, 065803

Asaoka, I., \& Aschenbach, B. 1994, A\&A, 284, 573

Asaoka, I., Egger, R., \& Aschenbach, B. 1996, Röntgenstrahlung from the Universe, eds. H. U. Zimmermann, J. Trümper, \& H. Yorke, 233

Aschenbach, B. 1998, Nature, 396, 141

Astropy Collaboration (Robitaille, T. P., et al.) 2013, A\&A, 558, A33

Audi, G., Bersillon, O., Blachot, J., \& Wapstra, A. H. 2003, Nucl. Phys. A, 729,

Badenes, C., Maoz, D., \& Draine, B. T. 2010, MNRAS, 407, 1301

Becker, W. 2009, Astrophys. Space Sci. Lib., 357, 91

Bertin, E., Mellier, Y., Radovich, M., et al. 2002, ASP Conf. Ser., 281, 228

Borkowski, K. J., Lyerly, W. J., \& Reynolds, S. P. 2001, ApJ, 548, 820

Busser, J.-U., Egger, R., \& Aschenbach, B. 1996, Röntgenstrahlung from the Universe, eds. H. U. Zimmermann, J. Trümper, \& H. Yorke, 239

Calabretta, M. R., Staveley-Smith, L., \& Barnes, D. G. 2014, PASA, 31, e007

Carretti, E., Haverkorn, M., Staveley-Smith, L., et al. 2019, MNRAS, 489, 2330

Case, G. L., \& Bhattacharya, D. 1998, ApJ, 504, 761

Chatterjee, S., Vlemmings, W. H. T., Brisken, W. F., et al. 2005, ApJ, 630, L61

Chen, J., Singh, B., \& Cameron, J. A. 2011, Nucl. Data Sheets, 112, 2357

Condon, J. J., Cotton, W. D., Greisen, E. W., et al. 1998, AJ, 115, 1693

Cordes, J. M., \& Lazio, T. J. W. 2002 ArXiv e-prints [arXiv:astro-ph/0207156]

Cordes, J. M., Romani, R. W., \& Lundgren, S. C. 1993, Nature, 362, 133

de Gasperin, F., Intema, H. T., \& Frail, D. A. 2018, MNRAS, 474, 5008

Dickey, J. M., \& Lockman, F. J. 1990, ARA\&A, 28, 215

Diehl, R., Siegert, T., Hillebrandt, W., et al. 2015, A\&A, 574, A72

Diehl, R., Siegert, T., Greiner, J., et al. 2018, A\&A, 611, A12

Dorman, B., Arnaud, K. A., \& Gordon, C. A. 2003, AAS/High Energy Astrophysics Division, 7, AAS/High Energy Astrophysics Division, 22.10

Dubner, G., \& Giacani, E. 2015, A\&ARv, 23, 3

Dubois, P. F., Hinsen, K., \& Hugunin, J. 1996, Comput. Phys. Commun., 10, 262

Ebeling, H., White, D. A., \& Rangarajan, F. V. N. 2006, MNRAS, 368, 65

Egger, R., Greiner, J., \& Aschenbach, B. 1996, Röntgenstrahlung from the Universe, eds. H. U. Zimmermann, J. Trümper, \& H. Yorke, 247

Faucher-Giguere, C.-A., \& Kaspi, V. M. 2006, ApJ, 643, 332

Fink, M., Röpke, F. K., Hillebrandt, W., et al. 2010, A\&A, 514, A53

Fink, M., Kromer, M., Seitenzahl, I. R., et al. 2014, MNRAS, 438, 1762

Folgheraiter, E. L., Watson, M. G., \& Warwick, R. S. 1996, Röntgenstrahlung from the Universe, eds. H. U. Zimmermann, J. Trümper, \& H. Yorke, 253

For, B. Q., Staveley-Smith, L., Hurley-Walker, N., et al. 2018, MNRAS, 480, 2743

Foster, A. R., Ji, L., Smith, R. K., \& Brickhouse, N. S. 2012, ApJ, 756, 128

Frail, D. A., Goss, W. M., \& Whiteoak, J. B. Z. 1994, ApJ, 437, 781

Gaia Collaboration (Brown, A. G. A., et al.) 2018, A\&A, 616, A1

Green, D. A. 2019, JApA, 40, 36

Green, D. A., \& Stephenson, F. R. 2017, Handbook of Supernovae, eds. A. W. Alsabti, \& P. Murdin (Berlin: Springer), 179

Gritschneder, M., Lin, D. N. C., Murray, S. D., Yin, Q. Z., \& Gong, M. N. 2012, ApJ, 745, 22

Halloin, H. 2009, Ispimodfitl Explanatory Guide and Users Manual, version 2.9 edn. (Garching: Max Planck Institut für extraterrestrische Physik)

Hancock, P. J., Trott, C. M., \& Hurley-Walker, N. 2018, PASA, 35, e011

Harris, C. R., Millman, K. J., van der Walt, S. J., et al. 2020, Nature, 585, 357

Hartmann, D. H., Predehl, P., Greiner, J., et al. 1997, Nucl. Phys. A, 621, 83

Haslam, C. G. T., Salter, C. J., Stoffel, H., \& Wilson, W. E. 1982, A\&AS, 47, 1

Hermansen, K., Couch, S. M., Roberts, L. F., Schatz, H., \& Warren, M. L. 2020, ApJ, 901, 77

Hindson, L., Johnston-Hollitt, M., Hurley-Walker, N., et al. 2016, PASA, 33, e020

Hunter, J. D. 2007, Comput. Sci. Eng., 9, 90

Hurley-Walker, N., \& Hancock, P. J. 2018, Astron. Comput., 25, 94

Hurley-Walker, N., Callingham, J. R., Hancock, P. J., et al. 2017, MNRAS, 464 1146
Hurley-Walker, N., Hancock, P. J., Franzen, T. M. O., et al. 2019a, PASA, 36, e047

Hurley-Walker, N., Filipović, M. D., Gaensler, B. M., et al. 2019b, PASA, 36, e045

Hurley-Walker, N., Gaensler, B. M., Leahy, D. A., et al. 2019c, PASA, 36, e048

Joye, W. A., \& Mandel, E. 2003, ASP Conf. Ser., 295, 489

Keane, E. F., \& Kramer, M. 2008, MNRAS, 391, 2009

Kochanek, C. S., Beacom, J. F., Kistler, M. D., et al. 2008, ApJ, 684, 1336

Kothes, R., Reich, P., Foster, T. J., \& Reich, W. 2017, A\&A, 597, A116

Leahy, D. A., \& Williams, J. E. 2017, AJ, 153, 239

Limongi, M., \& Chieffi, A. 2018, ApJS, 237, 13

Maeda, K., Röpke, F. K., Fink, M., et al. 2010, ApJ, 712, 624

Magkotsios, G., Timmes, F. X., Hungerford, A. L., et al. 2010, ApJS, 191, 66

Manchester, R. N., Hobbs, G. B., Teoh, A., \& Hobbs, M. 2005, AJ, 129 1993

Meidinger, N., Andritschke, R., Bornemann, W., et al. 2014, SPIE Conf. Se., 9144, 91441W

Meyers, B. W., Hurley-Walker, N., Hancock, P. J., et al. 2017, PASA, 34, e013

Moll, R., \& Woosley, S. E. 2013, ApJ, 774, 137

Nagataki, S., Shimizu, T. M., \& Sato, K. 1998, ApJ, 495, 413

Offringa, A. R., \& Smirnov, O. 2017, MNRAS, 471, 301

Offringa, A. R., McKinley, B., Hurley-Walker, N., et al. 2014, MNRAS, 444, 606

Offringa, A. R., Wayth, R. B., Hurley-Walker, N., et al. 2015, PASA, 32, e008

Offringa, A. R., Trott, C. M., Hurley-Walker, N., et al. 2016, MNRAS, 458, 1057

Oliphant, T. E. 2007, Comput. Sci. Eng., 9, 10

Pavlinsky, M., Levin, V., Akimov, V., et al. 2018, SPIE Conf. Ser., 10699. $106991 \mathrm{Y}$

Perets, H. B., Gal-Yam, A., Mazzali, P. A., et al. 2010, Nature, 465, 322

Pfeffermann, E., \& Aschenbach, B. 1996, Röntgenstrahlung from the Universe, eds. H. U. Zimmermann, J. Trümper, \& H. Yorke, 267

Pfeffermann, E., Briel, U. G., \& Freyberg, M. J. 2003, Nucl. Instrum. Methods Phys. Res. A, 515, 65

Predehl, P., Sunyaev, R., Becker, W., et al. 2020, Nature, 588, 227

Predehl, P., Andritschke, R., Arefiev, V., et al. 2021, A\&A, 647, A1 (eROSITA SI)

Remazeilles, M., Dickinson, C., Banday, A. J., Bigot-Sazy, M. A., \& Ghosh, T. 2015, MNRAS, 451, 4311

Reynolds, S. P., Borkowski, K. J., Green, D. A., et al. 2008, ApJ, 680, L41

Sault, R. J., Teuben, P. J., \& Wright, M. C. H. 1995, ASP Conf. Ser., 77, 433

Seitenzahl, I. R., Ciaraldi-Schoolmann, F., Röpke, F. K., et al. 2013, MNRAS, 429, 1156

Siegert, T., Diehl, R., Weinberger, C., et al. 2019, A\&A, 626, A73

Sokolowski, M., Colegate, T., Sutinjo, A. T., et al. 2017, PASA, 34, e062

Stephenson, F. R. 2017, Historical Records of Supernovae, eds. A. W. Alsabti, \& P. Murdin (Berlin: Springer), 49

Strong, A. W., Diehl, R., Halloin, H., et al. 2005, A\&A, 444, 495

Strüder, L., Briel, U., Dennerl, K., et al. 2001, A\&A, 365, L18

Su, H., Macquart, J. P., Hurley-Walker, N., et al. 2018, MNRAS, 479, 4041

Taylor, M. B. 2005, ASP Conf. Ser., 347, 29

Taylor, J. H., Manchester, R. N., \& Lyne, A. G. 1993, ApJS, 88, 529

Timmes, F. X., Woosley, S. E., Hartmann, D. H., \& Hoffman, R. D. 1996, ApJ, 464,332

Tingay, S. J., Goeke, R., Bowman, J. D., et al. 2013, PASA, 30, 7

Vedrenne, G., Roques, J.-P., Schönfelder, V., et al. 2003, A\&A, 411, L63

Voges, W., Aschenbach, B., Boller, T., et al. 1999, A\&A, 349, 389

Waldman, R., Sauer, D., Livne, E., et al. 2011, ApJ, 738, 21

Wanajo, S., Müller, B., Janka, H.-T., \& Heger, A. 2018, ApJ, 852, 40

Wayth, R. B., Lenc, E., Bell, M. E., et al. 2015, PASA, 32, e025

Wayth, R. B., Tingay, S. J., Trott, C. M., et al. 2018, PASA, 35, e033

Weinberger, C., Diehl, R., Pleintinger, M. M. M., Siegert, T., \& Greiner, J. 2020, A\&A, 638, A83

Wilms, J., Allen, A., \& McCray, R. 2000, ApJ, 542, 914

Winkler, C., Courvoisier, T. J.-L., Di Cocco, G., et al. 2003, A\&A, 411, L1

Woosley, S. E., \& Kasen, D. 2011, ApJ, 734, 38

Woosley, S. E., Arnett, W. D., \& Clayton, D. D. 1973, ApJS, 26, 231

Zimmermann, H. U., Becker, W., Belloni, T., et al. 1994, EXSAS User's Guide 